\title{
On Congruences Induced by Certain Relations on "Semigroups"
}

\author{
K. V. R. Srinivas \\ Department of Mathematics, Regency Institute of Technology, Yanam, India \\ Email: srinivaskandarpa73@gmail.com
}

Received 14 March 2015; accepted 28 July 2015; published 31 July 2015

Copyright (C) 2015 by author and Scientific Research Publishing Inc.

This work is licensed under the Creative Commons Attribution International License (CC BY). http://creativecommons.org/licenses/by/4.0/

(c) (i) Open Access

\begin{abstract}
In his paper "On quasi-separative 'semigroup's'", Krasilnikova, Yu. I. and Novikov, B. V. have studied congruences induced by certain relations on a "semigroup". They further showed that if the "semigroup" is quasi separative then the induced congruence is a semilattice congruence. In this paper we continue the study of these relations and the induced congruences i.e., the congruences induced by certain relations on "semigroup's". In this paper mainly it is observed that if $S$ is a quasi-separative and regular "semigroup" then the necessary and sufficient condition for $\overline{S \times S}$ to be the smallest semilattice congruence $\eta$ is obtained.
\end{abstract}

\section{Keywords}

Cancellative "Semigroup”, Quasi-Separative “Semigroup's”, Weakly Cancellative "Semigroup's”, Weakly Balanced "Semigroup"

\section{Introduction}

In this paper "On quasi-separative 'semigroup's”, Krasilnikova Yu. I. and Novikov B.V. have studied congruences induced by certain relations on a "semigroup". They further showed that if the "semigroup" is quasi-separative then the induced congruence is a semilattice congruence. In this paper we continue the study of these relations and the induced congruences. In theorem 2, we have proved that the family of all relatios $\mathscr{C}$ which satisfy the conditions from (1) to (3) in Def. 1 of this paper is a complete lattice. In theorem 3, we have also obtained that the family of all congruences which are induced by the relations in $\mathscr{H}$ is a complete lattice. If $S$ is a quasi-separative and regular "semigroup" then the necessary and sufficient condition for $\overline{S \times S}$ to be the smallest semilattice congruence which is denoted by $\eta$ (throughout this chapter) is obtained, from which as a corollary that if $S$ is a commutative regular "semigroup" then the congruence induced by the $S \times S$ is the smallest semilattice congruence [1]. The authors have remarked that a semilattice of weakly cancellative "semigroup's" 
is weakly balanced, it is not known that "whether semilattice of weakly cancellative 'semigroup's' [2] is weakly balanced", show that the result is not true. It is also observed that every semilattice of weakly cancellative "semigroup's", need not be weakly balanced, for this an example is obtained.

\section{Main Content}

The following definition is due to Krasilnikova Yu. I. and Novikov B. V. (see [3]).

Def 1: Let $S$ be a "semigroup" and $\Omega$ be a relation on $S$ satisfying conditions.

$$
\begin{aligned}
& \forall a \in S, \Omega \cap E(a)=\Omega \cap F(a) \rightarrow \\
& \forall a, b \in S, b(\Omega \cap E(a b)) \subseteq \Omega \rightarrow \\
& \forall a, b \in S,(\Omega \cap F(a b)) a \subseteq \Omega \rightarrow
\end{aligned}
$$

where $E(a)=\{(x, y) \in S \times S / a x=a y\}$ and $F(a)=\{(x, y) \in S \times S / x a=y a\}$

Define a relation $\bar{\Omega}$ on S corresponding to $\Omega$ by $\overline{a \Omega b}$ if and only if $\Omega \cap E(a)=\Omega \cap E(b)$. It is also equivalent to $\overline{a \Omega b}$ if and only if $\Omega \cap F(a)=\Omega \cap F(b)$, this relation $\bar{\Omega}$ is a congruence on $S$.

Lemma 2: Let $\mathscr{H}$ be the family of all relations on $S$ which satisfy the conditions from (1) to (3) then is a complete lattice.

Proof: Let $\mathscr{K}=\{\Omega \subset S \times S / \Omega$ satisfies from(1) to(3) in def1, of this paper $\}$. Then clearly $1_{s} \in \mathscr{K}$. Let $\left\{\Omega_{\alpha}\right\}$ be a subset of $\mathscr{H}$. Then both $\cup \Omega_{\alpha}$ and $\cap \Omega_{\alpha}$ are in $\mathscr{H}$. Therefore $\mathscr{H}$ is a complete lattice.

Lemma 3: Let $\overline{\mathscr{H}}=\{\bar{\Omega} / \Omega \in \mathscr{H}\}$ then $\overline{\mathscr{C}}$ is a complete lattice.

Proof: Since $1_{s} \in \mathscr{H} \quad \overline{1_{s}} \in \overline{\mathscr{H}}$. Therefore $\overline{\mathscr{H}} \neq \phi$. Also $\overline{1_{s}}$ is the greatest element in $\overline{\mathscr{K}}$. Let $\left\{\Omega_{\alpha}\right\}$ be a subset of. Then $\overline{\mathscr{H}} \cap \bar{\Omega}_{\alpha}=\cup \bar{\Omega}_{\alpha} \in \mathscr{H}$ because $=\cup \bar{\Omega}_{\alpha} \in \mathscr{H}$.

Theorem 4: Let $S$ be quasi-separative and regular "semigroup". Then $\overline{S \times S}=\eta$ if and only if for any $a, x, y \in S, a x=a y \Leftrightarrow x a=y a$.

Proof: Suppose $S$ is quasi-separative and regular and Suppose $\overline{S \times S}=\eta$. Then $S \times S \in \mathscr{H}$, so that it satisfies (1). Therefore $a x=a y$ if and only if $x a=y a$ for any $x, y \in S$. Conversely suppose that $a x=a y \Leftrightarrow x a=y a$. Then $S \times S \in \mathscr{H}$. Since $S$ is quasi-separative $\overline{S \times S}$ is semilattice congruence and hence $\eta \subseteq \overline{S \times S}$. Let $(a, b) \subseteq \overline{S \times S}$ so that $E(a)=E(b)$ and hence $a x=a y$ if and ony if $b x=b y$ for any $x, y \in S$, since $\mathrm{S}$ is regular there exists $a^{\prime}, b^{\prime} \in S$ such that $a=a a^{\prime} a$ and $b=b b^{\prime} b$. Put $e=a^{\prime} a$ and $f=b^{\prime} b$ then we have $a \cdot e=a$ and $b \cdot f=b$ so that $a e f=a f$ hence $b e f=b f$. Therefore $b a^{\prime} a f=b$ and similarly we have $a b^{\prime} b e=a$. Therefore if $F$ is any filter in $S$ then $a \in F$ if and only if $b \in F$ so that $(a, b) \in \eta$ and hence $\overline{S \times S}=\eta$.

Corollary 5: If $S$ is a commutative regular "semigroup" then $\overline{S \times S}=\eta$.

Corollary 6: If $S$ is a completely regular and $S \times S \in \mathscr{K}$ and if $\bar{\Omega}=\eta$, for some $\Omega \in \mathscr{K}$ then $S \times S=\eta$.

The following is an example of a completely regular "semigroup" in which $S \times S \notin \mathscr{K}$.

Example 7: Let $S$ be a left zero "semigroup" with at least two elements. If $S \times S \notin \mathscr{H}$ then $E(a)=F(a) \forall a \in S$ then $|S|=1$, which is a contradiction and hence $S \times S \notin \mathscr{H}$.

Theorem 8: In a band $S, S \times S \notin \mathscr{C} \quad$ if and only if $S$ is a semilattice.

It is natural to ask whether every semilattice congruence on "semigroup" is of the form $\bar{\Omega}$ for some $\Omega \in \mathscr{C}$.

The following example shows that it is not true.

Example 9: Consider the non modular lattice $L=(L, \wedge, \vee)$ in Figure 1 and let $S$ be the "semigroup" $(L, \wedge)$ is a semilattice. Clearly $F=\{a, b, 1\}$ is a filter in $L$ so that $\theta_{F}=\{(a, b) \mid a \wedge f=b \wedge f$ for some $f \in F\}$ is a congruence on $S$. But $\theta_{F} \neq \bar{\Omega}$ for any $\Omega \in \mathscr{H}$. 


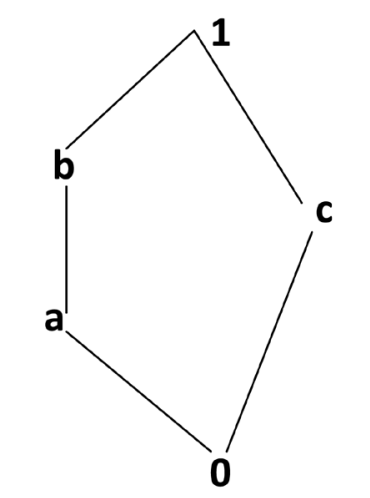

Figure 1. $a^{c}=b^{c}=0$, take $c=f$.

The following example shows that $\bar{\Omega}$ is a semi lattice congruence whenever $\Omega \in \mathscr{K}$ then the "semigroup" need not be quasi-separative.

Example 10: Let $S=\{0, a\}$ be two element null "semigroup". Clearly $\mathscr{K}=\left\{1_{S}, S \times S\right\}$ and $1_{S}=\overline{S \times S}=S \times S$ which is a semilattice ongruence. But is not a quasi-separative (since $a^{2}=a \cdot 0=a^{2}$ and $a \neq 0$ ).

The following example shows that in non quasi-separative "semigroup's” there exists $\Omega \in \mathscr{K}$ such that $\bar{\Omega}$ is a semi-lattice congruence.

Example 11: Let $S$ be a non quasi-separative "semigroup", then $1_{S}$ is in $\mathscr{H}, 1_{S}=\overline{S \times S}$ and $S \times S$ is a semi-lattice congruence.

It is interesting to note that if $S$ is a left or right zero "semigroup" then

$$
\mathscr{H}=\left\{1_{S}\right\} .
$$

In paper [1] they have remarked that it is not known that whether semilattice of weakly cancellative "semigroup's" is quasi-separative and weakly balanced. In the following we are giving an example which shows that it is not true i.e. if a "semigroup's" is isomorphic to a semilattice of weakly cancellative "semigroup's" then $S$ is a quasi-separative and weakly balanced.

Example 12: Consider the "semigroup" $S=\{a, b, c, d\}$ with multiplication table as follows:

\begin{tabular}{lllll}
\hline$\cdot$ & $a$ & $b$ & $c$ & $d$ \\
$a$ & $a$ & $b$ & $a$ & $a$ \\
$b$ & $a$ & $b$ & $b$ & $b$ \\
$c$ & $a$ & $b$ & $c$ & $d$ \\
$d$ & $a$ & $b$ & $c$ & $d$ \\
\hline
\end{tabular}

Then $\eta$-classes are $\{a, b\}$ and $\{c, d\}$ which are right zero "semigroup's" and hence $S$ is a semilattice of weakly cancellative "semigroup's", but $S$ is not weakly balanced since $a \cdot a=a \cdot c, a \cdot b=c \cdot b$, but $b \cdot a \neq b \cdot c$.

The following is an example of quasi separative "semigroup", which is not completely regular.

Example 13: Consider the "semigroup" $S=N \times N$ on which "." is defined by

$$
(m, n) \cdot(p, q)=(m-n+t, q-p+t)
$$

where $t=\max \{n, p\}$. Clearly $S$ is quasi separative "semigroup", and since the inverse of $(m, n)$ is $(n, m)$ and $(m, n) \cdot(n, m) \neq(n, m) \cdot(m, n), S$ is not completely regular.

Thorem 14: Let $S$ be a separative "semigroup", and $a \in S$ such that $E(a)$ is a semilattice congruence then $a \in E(S) \cap C(S)$.

Proof: Let $S$ be a separative "semigroup" and $a \in S$ such that $E(a)$ is a semilattice congruence. Then for any 
$x, y \in S, \quad(x y, y x) \in E(a)$, so that $a x y=a y x \quad \forall x, y \in S$. Now replace " $y$ " by " $b$ " and " $x$ " by " $a$ " then $a \cdot a b=a \cdot b a \forall b \in S$ which implies $b a \cdot a b=b a \cdot b a$. Since $S$ is separative and $a^{2} b=a b a$ we have $b a^{2}=a b a$ so that $b a \cdot a b=(a b)^{2}$. Since $S$ is separative we have $a b=b a$. Again since $E(a)$ is a semilattice congruence, $\left(a, a^{2}\right) \in E(a)$ so that $a^{2}=a$ and hence $a \in E(S) \cap C(S)$.

\section{Acknowledgements}

We are very much thankful to the referees for their valuable suggestions.

\section{References}

[1] Burmistrovich, I.E. (1965) Commutative Bands of Cancellative “Semigroup’s””. Sib.Mat.Zh., 6, 284-299 (Russian).

[2] Petritch, M. (1973) Introduction to “'Semigroup’ Theory”. Merill Books, Columbus.

[3] Krasilnikova, Yu.I. and Novikov, B.V. (2005) On Quasi-separative “semigroup’s”. “Semigroup” Forum, 70, $347-355$. 